ÉGYPTE monde arabe

\section{Égypte/Monde arabe}

30-31 | 1997

Les visions de l'Occident dans le monde arabe

\title{
« Vent d'est, vent d'ouest » : l'Occident du droit égyptien
}

\section{Baudouin Dupret}

\section{(2) OpenEdition}

1 Journals

Édition électronique

URL : https://journals.openedition.org/ema/1603

DOI : 10.4000/ema.1603

ISSN : 2090-7273

\section{Éditeur}

CEDEJ - Centre d'études et de documentation économiques juridiques et sociales

\section{Édition imprimée}

Date de publication : 30 septembre 1997

Pagination : 93-111

ISSN : 1110-5097

\section{Référence électronique}

Baudouin Dupret, « « Vent d'est, vent d'ouest » : I'Occident du droit égyptien », Égypte/Monde arabe [En ligne], 30-31 | 1997, mis en ligne le 08 juillet 2008, consulté le 07 juillet 2022. URL : http:// journals.openedition.org/ema/1603 ; DOI : https://doi.org/10.4000/ema.1603

Ce document a été généré automatiquement le 7 juillet 2022.

Tous droits réservés 


\title{
« Vent d'est, vent d'ouest »: l'Occident du droit égyptien
}

\author{
Baudouin Dupret
}

1 Je pars dans cet article d'une double interrogation. S'il est vrai, comme l'affirme Nathan Brown (1995), que le droit égyptien contemporain et sa progressive élaboration ne peuvent être considérés sous l'angle du seul instrument de la domination impérialiste ; et s'il est vrai que le rôle des Égyptiens eux-mêmes - et surtout de leurs élites - ne saurait être négligé dans le façonnage d'un système juridique susceptible d'assurer la construction de l'État et la défense contre l'impérialisme, il reste toutefois légitime de se demander pourquoi c'est au système juridique occidental qu'il a été fait appel. En d'autres termes, s'il n'y a pas eu de volonté extérieure tendant à imposer ce modèle, pourquoi s'imposait-il de l'adopter? Se posent ainsi la question de savoir comment le système juridique occidental en est arrivé à constituer un modèle et, partant, la question de son efficacité - éventuellement présumée -, et du caractère inapproprié du système juridique existant qu'il était supposé venir corriger. Se pose ensuite la question des modalités du transfert juridique. L'opération a-t-elle consisté en un changement de paradigme, au sens où Kuhn l'entend, correspondant à une véritable révolution dans la "science du droit », ou bien, au contraire, s'est-elle inscrite dans un contexte fait de postulats normatifs communs aux deux univers juridiques et facilitant les transferts de l'un à l'autre?

2 Mon objectif, dans cet article, n'est pas d'ordre historique. Il ne s'agit pas de retracer les voies par lesquelles le système juridique égyptien s'est transformé pour devenir ce qu'il est aujourd'hui. Par contre, je souhaiterais démontrer que l'instrumentalisation du droit - en l'espèce, l'utilisation du modèle juridique occidental aux fins de combattre l'impérialisme - ne saurait être confondue avec son « égyptianisation » - le procédé par lequel le modèle d'importation se verrait réinjecter les caractéristiques fondamentales de la «culture » juridique locale. Autrement dit, le fait que le modèle juridique occidental ait été utilisé par l'élite égyptienne pour se défendre de la domination occidentale ne signifie pas pour autant que ce modèle ait été l'objet d'un processus tendant à réintroduire les caractères et exigences de la société égyptienne, 
mais seulement qu'il a constitué l'instrument d'un projet spécifique. Instrument d'une élite dans sa résistance à l'hégémonie d'un pouvoir étranger, il est aussi progressivement devenu l'instrument de cette élite dans la consolidation de son pouvoir et de sa politique de modernisation autoritaire. Je considère donc que l'adaptation du droit d'inspiration occidentale au contexte égyptien ne doit pas être surestimée.

3 D'abord, parce qu'il y a bien eu transfert. Celui-ci peut être rapidement attesté historiquement. Au niveau de la jurisprudence actuelle, cela se vérifie, entre autres, dans le fait qu'on retrouve dans le droit égyptien une série de postulats directement empruntés au modèle juridique d'importation - français essentiellement.

Ceci étant, ce transfert a pris souche et il faut l'expliquer. La première explication tient aux conditions du recours au modèle occidental. L'effet de contrainte coloniale n'a peut-être pas été direct, mais ce n'est cependant pas en dehors de tout rapport de force asymétrique que l'opération a été menée. Si l'on se pose, par ailleurs, la question de la place et de la fonction occupée par la sharî'a dans le processus de transfert, il faut relever, à un niveau plus structurel, une homologie des systèmes normatifs juridique et religieux. Dans ce contexte, il convient alors de souligner le rôle joué par la sharî'a, non pas en tant que système juridique importateur, mais en tant que "postulat d'identité du droit indigène », pour reprendre l'expression de Massaji Chiba.

\section{Transferts juridiques et transformations du droit}

5 Dans cette première partie, je voudrais m'attacher, d'une part, à rappeler les grandes lignes et étapes des transferts juridiques opérés en Égypte contemporaine et, d'autre part, à mettre en exergue, par le recours à des extraits de jurisprudence, la présence massive dans l'ordre juridique égyptien des postulats propres à sa source française.

\section{Quelques repères historiques}

Quelques rappels historiques très rapides devraient aider à resituer les modalités et conditions de réception d'un modèle occidental de formulation du droit et d'administration de la justice. On peut faire remonter à 1876 (date de création des tribunaux mixtes, dotés de codes conçus par Maunoury, juriste français d'Alexandrie) et 1883 (date de création des tribunaux indigènes, dotés de codes rédigés par l'Italien Moriondo et le Français Vacher de Montguyon) le moment de l'entrée effective de l'Égypte dans la famille du droit français (Goldberg, 1997). Cromer lui-même reprocha aux nouveaux codes de n'être pas suffisamment « égyptiens » (Cromer, 1911, p. 708, cité par Goldberg, 1997). On peut donc penser que le droit français s'était bien implanté dans le pays. Le processus plonge sans doute ses racines dans l'occupation française de l'Égypte (1798-1801), même si ce n'est pas de cette époque que datent les réformes importantes.

7 Le XIXe siècle a vu se multiplier les efforts des gouverneurs (formellement) ottomans, vice-rois et khédives, pour faire prendre au système juridique et judiciaire une coloration "moderne », c'est-à-dire avant tout occidentale (Hill, 1987; Reid, 1981 ; Ziadeh, 1968 ; Botiveau, 1989) ${ }^{1}$. L'introduction des changements à la fois juridiques et judiciaires débuta sous le règne de Muhammad 'Alî, pour se poursuivre avec ses successeurs. Le moyen utilisé à cette fin fut celui du qânûn, terme à comprendre pour 
cette époque par règlement administratif. C'est ainsi que furent établis de nouveaux ministères, des conseils aux compétences judiciaires et des tribunaux, à la tête desquels une Haute Cour pour les affaires civiles (majlis al-ahkâm). De même, de nouvelles lois furent promulguées. La progressive sujétion de l'Égypte aux contraintes du commerce international et de l'impérialisme occidental déboucha sur l'instauration de "Tribunaux de marchands » utilisant législation et avocats français. Des «Tribunaux mixtes" (mahâkim mukhtalata), puis des "Tribunaux indigènes" (mahâkim ahliyya) furent ensuite créés. Parallèlement, les Tribunaux de la shari'a continuaient de fonctionner pour les questions touchant au statut personnel et, au début, pour les matières pénales. Ces tribunaux furent toutefois progressivement dépouillés de leurs compétences pour finalement être absorbés, en 1956, par le système des Cours et Tribunaux nationaux. Notons aussi la fondation, en 1946, d'un Conseil d'État (Majlis aldawla) calqué sur le modèle français, selon les termes mêmes d'un de ses présidents, 'Abd al-Razzâq al-Sanhûrî, ou encore, d'une Cour constitutionnelle (Mahkama dustûriyya 'ulyâ) en 1979 (Jacquemond, 1988).

8 En matière de droit positif, la première idée fut de codifier la sharî́a à l'image de la Mecelle ottomane de 1869-1876, ce qui explique le travail entrepris par Qadrî Pacha (ministre de la Justice de 1879 à 1882), dont l'oeuvre la plus connue est le

9 Murshid al-hayrân portant sur le droit civil et le droit des contrats. Certains éléments permettent d'affirmer qu'existait à l'époque la conviction qu'il fallait structurer les tribunaux indigènes de telle façon que «les Européens puissent s'y fier », ce qui allait de pair avec une mise en question de l'opportunité d'une codification de la sharî'a et de son adéquation aux coutumes et transactions des gens, qu'ils soient égyptiens ou étrangers. Les Tribunaux mixtes restèrent longtemps en activité et leurs codes furent très largement reproduits à l'intention des juridictions nationales, ce qui explique que le droit civil égyptien ait été composé de lois calquées sur le modèle français et reproduisant la conception française du droit. Ce phénomène fut de plus renforcé par le rôle majeur exercé par les juges d'origine européenne qui occupèrent longtemps (jusqu'à la Convention de Montreux de 1937) les sièges des Tribunaux mixtes.

10 Ce phénomène de transfert technologique s'est traduit également par l'apparition d'une catégorie de professionnels du droit égyptiens, de même que par le développement d'un enseignement du droit dit moderne. C'est ainsi que la proportion des juges égyptiens au sein des Tribunaux mixtes alla grandissant. De nouveaux vocables apparurent, comme celui d'«avocat " (muhâmî, qui semble avoir été utilisé pour la première fois en 1888 dans un texte réglementant les juridictions nationales (Botiveau, 1993, p.161-164), vocables qui correspondent à l'émergence de nouvelles fonctions. Les associations professionnelles leur correspondant apparurent dans la foulée (i.e., le Syndicat des avocats en 1912). L'enseignement du droit moderne fut, pour sa part, d'abord le fait de l'École des langues (madrasat al-alsun), fondée en 1836 et un temps dirigée par le très célèbre réformiste Rifâ'a Râfi' al-Tahtâwî, fermée sous le règne du khédive 'Abbâs puis réouverte en 1968 sous le nom d'École d'administration et des langues (madrasat al-idâra wa-l-alsun), enfin renommée École de droit (madrasat al-huqûq) en 1886. Cette institution fut intégrée à l'Université du Caire naissante, en 1925, en tant que Faculté de droit. L'École de droit eut à sa tête, jusqu'en 1907, des directeurs français (le dernier étant Édouard Lambert, comparatiste célèbre de l'Université de Lyon). En dépit de la présence anglaise toujours plus marquée, l'influence française perdura, du fait de la nature même du système juridique mis en place et aussi par le biais de l'École 
française de droit ouverte en 1890. De plus, une véritable tradition de séjour doctoral en France fut inaugurée à cette époque (sans parler de l'expérience précédente de Tahtâwî), et se prolonge jusqu'aujourd'hui. Les personnalités les plus fameuses du monde juridique égyptien sont presque toutes sorties de ce moule. Ainsi de Sanhûrî, le véritable concepteur du Code civil égyptien (al-Wasîf): diplômé de l'École de droit, procureur et un temps juge du Tribunal mixte de Mansûra, il soutiendra au début des années vingt sa thèse de doctorat sous la direction d'Édouard Lambert à Lyon, avant de rejoindre la Faculté de droit de l'Université du Caire à son retour de France, d'en devenir le doyen en 1936, et d'accéder à la présidence du Conseil d'État en 1949.

\section{Le transfert des postulats interprétatifs}

11 Je voudrais maintenant montrer que le transfert de technologie juridique est, en luimême, susceptible de rendre compte d'un processus de transfert des postulats à la base du système " exportateur » vers le système "importateur ", ceci ne préjugeant en rien des questions d'intériorisation desdits postulats par les acteurs du droit étatique ${ }^{2}$, qu'il $^{2}$ s'agisse des professionnels de ce droit ou de ceux qui sont amenés à y recourir.

12 Ainsi, quand il se réfère à la sharî'a, le juge égyptien contemporain se situe au croisement de deux types d'énonciation normative (le « dire le droit » du juge moderne issu de la Révolution et le "dire le prescrit de Dieu» du juge islamique). Pareille référence n'a rien d'exceptionnel et le droit positif ne semble pas fort s'émouvoir du recours à l'islam et à ses dispositions normatives, pourvu que cela ne se fasse pas sur la base d'une contestation de sa validité et/ou d'une exigence de soumission à un ordre qui lui est extérieur (Dupret, 1995). La production d'un certain nombre d'exemples concrets, tirés de l'une ou l'autre décision de justice rendue en Égypte, devrait permettre de confirmer notre hypothèse, à savoir que le système égyptien ne repose pas sur des fondements diamétralement opposés au système judiciaire moderne qui s'est développé en Occident et qu'il est donc passible d'une mise en perspective comparable.

13 S'il ne faut pas sous-estimer le processus de «naturalisation » égyptienne qui a traversé ce droit et ses institutions judiciaires, je ne suivrai pas pour autant Bernard Botiveau quand il affirme le caractère purement national de la jurisprudence ${ }^{3}$. Même "naturalisée », même si elle a «fait souche», cette "transplantation» du droit occidental, et plus particulièrement du droit de tradition latine, lui conserve l'essentiel des caractères dont il est revêtu dans son espace d'origine. En tête desquels ses postulats...

14 Le culte de la loi comme production normative exclusive transparaît constamment dans les jugements et arrêts rendus par les Cours et tribunaux égyptiens comme dans l'action et les propos des juristes de manière générale. C'est presque, oserions-nous dire, au nom de ce nomo-centrisme que le droit positif se voit invalider dans certains cas d'espèces. Ce n'est pas en effet la notion même de loi qui se trouve mise en cause ni le principe du législateur, mais uniquement la source de cette loi et l'identité de son législateur.

15 «Le juge, dans son jugement, exécute la règle de Dieu ou de Son Envoyé - que Dieu le bénisse et lui donne la paix -, non pas la règle de l'imam. Dès lors que les lois sont devenues concordantes et que les textes du Livre et de la Sunna sont allés de pair avec les principes généraux de la sharî̀a islamique et avec son esprit législatif, il est devenu 
obligatoire de leur obéir [...]. Mais lorsqu'elles se distinguent des principes Légaux, elles tombent sous le coup d'une nullité absolue et personne ne doit leur obéir. Il convient, au contraire, que tout juge musulman les combatte sans indulgence et qu'il s'institue lui-même leur ennemi.» (Tribunal d'arrondissement de Bulâq Abu al-'Alâ', 30 janvier 1982, cité par Ghurâb, 1986)

D'autres extraits significatifs peuvent être proposés. C'est ainsi qu'on retrouve l'idée d'un législateur constitutionnel doté d'une volonté unique - idée déduite de l'examen du texte de la Constitution.

«Considérant que l'article 56 de la Constitution dispose : [...]

«Il résulte de ce texte, qui figure au chapitre sur «les libertés, droits et devoirs publics», que le législateur constitutionnel ne s'est pas contenté, comme dans les Constitutions précédentes, de garantir le droit de former des syndicats jouissant de la personnalité morale (article 55 de la Constitution de 1956 et article 41 de la Constitution de 1964), mais a encore affirmé le principe de la démocratie syndicale en décidant que la formation des syndicats et fédérations repose sur une base démocratique, afin d'approfondir le régime démocratique adopté par la Constitution, qui est en fait la base fondamentale de l'État, comme le montre son article $1 \mathrm{er}[. .$.$] et de nombreux autres articles [...]$

"Considérant qu'il résulte de ce qui précède que le législateur constitutionnel, en disposant dans l'article 56 de la Constitution que « la création des syndicats et des fédérations sur une base démocratique est un droit garanti par la loi ", a voulu en fait affirmer le principe de la liberté syndicale dans sa conception démocratique, laquelle implique [...]» (HCCE, 1983, p. 563)

17 Quant à l'organisation du système juridique dans son ensemble, elle répond à des principes hiérarchiques rigoureusement établis, avec la Constitution au sommet d'une pyramide légale -assez naturellement considérée par le juge constitutionnel dans une perspective kelsénienne.

« Considérant que les textes constitutionnels sont l'interprétation des principes et des fondements du pouvoir dans l'État et qu'ils doivent prévaloir sur toute autre règle de droit, leur caractère obligatoire et le respect qui leur est dû découlent de cette suprématie. C'est à ces principes que l'on se réfère pour fixer les compétences générales des pouvoirs publics et toutes compétences spéciales dérogeant au principe général selon lequel ces autorités doivent limiter leur activité au domaine correspondant à la nature de leur fonction. La Constitution ayant énoncé limitativement ces compétences exceptionnelles, aucune autorité publique ne peut les outrepasser, sauf à commettre une infraction à la Constitution, [infraction] qui relève alors du contrôle juridictionnel exclusif de la Haute Cour constitutionnelle à laquelle cette même Constitution a confié le soin de sauvegarder les principes qu'elle a édictés. » (HCCE, 1985)

Les présupposés de complétude, de cohérence et d'unité de sens qui, historiquement, se sont d'abord attachés au texte avant d'être transposés au système, présupposés mis en évidence par Lenoble et Ost (1980), jalonnent le libellé de nombre de décisions et d'arrêts. La Constitution est ainsi marquée du sceau de la rationalité qui implique, par exemple, qu'elle ne formule pas une seule règle en deux dispositions.

«Dès lors, l'examen auquel procède l'Assemblée consultative sur les questions définies par l'article 194 de la Constitution, conduit à examiner en profondeur tous les différents aspects pour en arriver au fond de la question, ceci après un examen auquel l'Assemblée a procédé en en ayant généralement eu l'initiative et non en y étant contrainte par une partie quelconque qui l'en aurait requise. Elle décide également de la sorte de ses priorités dans son domaine et elle est libre de l'évaluation qu'elle estime justifiée et de ses chances réelles d'être efficace, dans la mesure où les moyens qui sont à sa disposition ont pour but de résoudre ces 
difficultés et de parvenir à leur solution effective [...]. Par ailleurs, elle ne considère pas la soumission de n'importe quelle question connexe comme une contrainte découlant de la Constitution. Preuve en est que la Constitution veille à ce qu'il soit distingué de manière absolue entre la fonction de l'Assemblée en vertu de l'article 194, d'un côté, et le pouvoir dont elle est investie dans le cadre de l'article 195, d'un autre côté, ce qui signifie que ces deux dispositions ne peuvent être confondues et qu'on ne peut admettre le propos visant à les confondre. Si cela avait été autorisé, la Constitution les aurait unifiées en une seule disposition. » (HCCE, 1993)

De manière semblable, le Constituant agit de manière cohérente dans l'établissement de conditions à l'exercice du pouvoir de légiférer par voie de décret dans les cas relevant de circonstances exceptionnelles.

"Considérant que le pouvoir de légiférer appartient à l'organe législatif représenté, aux termes de l'article 86 de la Constitution, par l'Assemblée du Peuple et qu'il doit être exercé par cet organe lui-même, conformément à la Constitution. Que cependant peuvent survenir, en l'absence de l'Assemblée du Peuple, des circonstances exigeant que soient prises sans délai des mesures appropriées à propos desquelles la Constitution autorise le président de la République à prendre des décrets ayant force de loi. Que le Constituant a tenu à ce que ce pouvoir législatif exceptionnel soit assorti de restrictions et de conditions telles qu'il ne puisse se transformer en pouvoir absolu de légiférer; que, ce faisant, il a tenu compte à la fois du principe de séparation des pouvoirs, qui garantit l'exercice direct par chacun de ces pouvoirs de leurs compétences restrictives, et de considérations pratiques découlant de l'urgence et exigeant qu'en l'absence de l'Assemblée législative normalement compétente, le président de la République soit autorisé, pour leur faire face - et à titre exceptionnel -, à légiférer. » (HCCE, 1985, p. 104-105)

On retrouve le postulat de cohérence dans l'adoption, par le juge constitutionnel, du principe de non-rétroactivité de l'amendement constitutionnel faisant de la sharî́a la source de la législation.

« Il résulte de ce qui précède que, depuis le 22 mai 1980, date d'entrée en vigueur de l'article 2 modifié de la Constitution, le pouvoir législatif est contraint de veiller à ce que les lois qu'il élabore - textes nouveaux ou réformes de textes antérieurs à cette date - soient conformes aux principes de la sharî'a, et que pour autant elles n'outrepassent pas les freins et limites imposés par la Constitution au législateur dans l'exercice de son pouvoir. Ce sont ces freins et limites, y compris ceux de l'article 2 modifié, qui délimitent le cadre du contrôle exercé par la Haute Cour constitutionnelle. Cependant, l'obligation faite au législateur de prendre les principes de la sharî'a comme source principale de la législation telle qu'exposée cidessus ne s'étend qu'aux textes légaux promulgués après la date de son entrée en vigueur, et tout texte de loi qui, dans ce cas, serait contraire aux principes de la sharî'a surviendrait en violation de la Constitution. Quant aux lois antérieures à cette date, elles ne peuvent pour cette seule raison être soumises à cette obligation, et se trouvent par-là même hors d'atteinte du contrôle de constitutionnalité qui en découle. Ce point de vue est conforté par le rapport présenté par la commission générale à l'Assemblée du Peuple et approuvé par elle le 15 septembre 1981 :

«La Constitution de 1971 était, dans notre histoire moderne, la première à prévoir expressément que la sharî́a islamique est une source principale de la législation; puis est venue la réforme de 1980, qui en a fait la source principale de la législation. Cela signifie que dorénavant aucune législation contraire aux commandements de la sharîa islamique ne peut être promulguée et qu'il est devenu nécessaire de réexaminer les lois antérieures à la Constitution de 1971 et sa réforme et de les amender afin de les rendre conformes aux commandements de la sharî'a islamique [...]. La transition du régime juridique actuellement en vigueur en Égypte, qui 
remonte à plus d'un siècle, au régime islamique intégral demandera du temps et de la réflexion. » (HCCE, 1985, p. 571)

21 Il est amusant de noter qu'à l'égard de cet article 2 de la Constitution, le débat s'est développé autour de la question de savoir si le postulat de solidarité (et donc de cohérence) des articles de la Constitution était respecté (Abu Tâlib, 1994) ou non (Botiveau 1993, p. 286), alors que notre propre démarche conduit à l'identification des postulats interprétatifs en tant qu'ils sont à la base d'une " dérive mytho-logique ». En fait, plus qu'un sujet d'amusement, cette remarque rappelle avec force que les tenants d'une «islamisation» du droit fonctionnent, pour nombre d'entre eux, de l'intérieur même d'un système juridique héritier des postulats du système « exportateur » et qu'ils n'entendent pas forcément s'opposer, dans la formulation de leurs revendications, à ce qui apparaît comme des principes constitutifs de ce dernier.

On peut aisément imaginer que ce que Lenoble et Ost décrivent, dans le contexte du droit de tradition latine, comme l'inféodation du juge à la loi par le jeu de la fiction de l'unité textuelle, puis de l'unité systémique (via l'image du législateur rationnel), trouve également à se réaliser dans le droit égyptien dont on extrait ici rapidement quelques formules symptomatiques. Dans le travail d'interprétation judiciaire qui s'effectue en Égypte, le « comme si » de la rationalité du législateur semble fonctionner à plein, avec les mêmes contraintes discursives tenant au texte, à l'auteur et à l'interprétation. Si l'on examinait les contraintes de nature plus spécifiquement juridique, l'on retrouverait assurément un ensemble de modèles normatifs d'interprétation. Par ailleurs, il est possible de pointer l'existence d'un rapport très étroit entre, d'une part, établissement, interprétation et qualification du fait, et, d'autre part, choix, interprétation et application de la disposition pertinente. La théorie d'une activité judiciaire en prise immédiate avec le « réel » transparait de manière récurrente. Ainsi en va-t-il de l'idée que le fait est présenté au juge dans sa réalité.

«Attendu que les pièces de la requête apparaissent sous la forme suivante :

«[...] Attendu que, dans l'accusation formulée sous cette forme, les éléments sont concordants à l'égard du coupable qui ne s'est pas défendu de l'accusation à l'aide d'un argument ou d'un moyen de défense jugé acceptable ou admissible par le tribunal et que, dès lors, sa punition s'impose.» (Tribunal d'arrondissement de Bulâq, 30 janvier 1982, cité dans Ghurâb, 1986)

Ainsi en va-t-il également de l'affirmation de l'intelligibilité et de la transparence du langage juridique, avec leurs corollaires de sens clair et de sens usuel, ainsi que d'une application non distordue du droit au fait. Un extrait d'arrêt de la Haute Cour constitutionnelle devrait permettre de s'en convaincre :

"Attendu qu'il ressort à l'évidence de ces deux dispositions que leur principe établit l'autorité de l'Assemblée consultative sur les questions de garantie de la préservation des révolutions du 23 juillet 1952 et du 15 mai 1971, ainsi que sur les questions liées à la consolidation de l'unité nationale, à la préservation de la paix civile, à la protection de l'alliance des forces actives du peuple et de leurs acquis socialistes, à l'ancrage des composantes fondamentales de la société et de ses valeurs supérieures, en ce compris les droits du citoyen, ses libertés, l'accomplissement de ses obligations générales, l'approfondissement du régime socialiste démocratique et l'élargissement de ses domaines d'action. L'intérêt de l'Assemblée se limite, sur l'ensemble de ces questions, à leur examen, en connaissance de cause et en ayant en mémoire les différents points de vue y afférents, ainsi qu'à leur présentation étayée par la présentation d'arguments apparaissant eux-mêmes valides et susceptibles de réaliser l'intérêt général dont la Constitution vise la préservation. N'y rentre pas et n'a pas vocation à y rentrer, 
dans le contexte de l'examen des questions déterminées par l'article 194 de la Constitution, la simple collaboration de l'État, au travers de ses différents pouvoirs et de ses multiples institutions, sous réserve qu'il prenne ses décisions en la matière en en ayant cerné tous les aspects et conscient de son impact positif et de ses répercussions négatives, tout ceci dans un cadre d'objectivité parfaite et à l'abri de tout glissement ou de penchant pour un point de vue qui ne serait pas conforté par les vérités pratiques. Dès lors, l'examen auquel procède l'Assemblée consultative sur les questions définies par l'article 194 de la Constitution conduit à pénétrer profondément tous les différents aspects pour en arriver au fond de la question, ceci après un examen auquel l'Assemblée a procédé en en ayant généralement eu l'initiative et non en y étant contrainte par une partie quelconque qui l'en aurait requise.» (HCCE, 1993)

Dans un autre arrêt, si l'argument du requérant doit être écarté, c'est en vertu du fait suivant :

«Si le législateur constitutionnel avait voulu faire des principes de la sharî'a islamique des règles constitutionnelles ou s'il avait entendu les rendre directement applicables par les tribunaux sans qu'il soit besoin de les développer dans une législation précise et conforme aux procédures prévues par la Constitution, il aurait fallu qu'il le stipule expressément. » (HCCE, 1985, p. 571)

Il est également affirmé, dans le même arrêt :

«L'argument du requérant [...] doit être écarté conformément au sens véritable de la réforme tel que l'a exposé la Cour ci-avant. » (HCCE, 1985, p. 571)

L'on peut aussi donner de multiples exemples du caractère opérant des valeurs juridiques. On se contentera d'un extrait pour illustrer les valeurs de sécurité, de stabilité et d'ordre.

«L'argument du requérant, s'il était retenu, aboutirait non seulement à abroger toutes les législations civiles, pénales, sociales et économiques ou autres contraires aux principes de la sharî'a, mais encore à contraindre les tribunaux à appliquer aux litiges qui leurs sont soumis des règles non codifiées à la place des lois abrogées, avec tous les risques de contradiction entre ces règles et de déstabilisation de l'ordre juridique que cela comporte. » (HCCE, 1985, p. 571)

Tous les éléments du modèle descriptif théorique proposé par Lenoble et Ost semblent donc bien devoir se retrouver, d'une manière ou d'une autre, dans le travail interprétatif du juge égyptien (dans la plupart des cas) qu'on a tenté d'illustrer succinctement. On croit en tout cas pouvoir y repérer des éléments tenant d'une précompréhension, d'une interprétation fondatrice, d'une interprétation effectuante et d'une vérification, avec les conséquences qui en ont été tirées sur le plan des fonctions d'argumentation, de raréfaction et de légitimation.

\section{Pourquoi le transfert a-t-il fait souche?}

Je voudrais à présent poser la question des mécanismes ayant assuré le succès de l'opération de transfert juridique que je pense avoir suffisamment attestée dans la première partie. Je fonderai l'explication en trois points. Il y a, d'abord, le constat de l'asymétrie des relations unissant deux contextes socio-politiques. Il faut ensuite faire le constat d'une homologie de structure des systèmes juridiques d'exportation et d'importation, entre eux et avec le système religieux. Enfin, il convient de s'interroger sur la place du droit d'inspiration religieuse (qu'on appellera par commodité sharî'a) et sur sa fonctionnalité dans le mécanisme d'importation. 


\section{Asymétrie politique et transferts juridiques} par une explication exclusivement centrée sur la domination coloniale. Celle-ci a bien exercé un effet sur les conceptions juridiques des élites politiques ayant présidé à l'élaboration du « droit moderne » et aux transferts dont il procède ; on ne saurait pour autant considérer qu'elle a purement et simplement imposé un ordre juridique exogène à des populations réfractaires. Bien au contraire, il convient de constater à quel point le processus d'importation et de transformation a été voulu et conçu par une élite politique « locale » qui, loin d'entériner par là sa soumission politique, a cherché à en faire l'instrument de sa résistance à la domination étrangère et de son projet nationaliste autoritaire. Je me montrerai pour ma part plus réticent sur le pas supplémentaire que franchit Brown quand il affirme que ce processus ne procède que réactivement du rapport de domination coloniale. Je pense - comme il le suggère, mais trop discrètement (Brown, 1995, p. 105) - que ce n'est pas uniquement pour résister à la présence coloniale qu'on a recouru à un système juridique d'inspiration occidentale, mais aussi en conséquence de cette présence qui a tendu à faire accréditer l'idée de l'inadéquation du droit local et de la nécessité de le conformer à un modèle caractérisé par son efficacité. En un mot, c'est la présence coloniale qui a conduit à considérer le système en place comme insuffisant et le système occidental comme nécessaire.

Il reste à se demander pourquoi ces élites ont jugé opportun de recourir au transfert de technologie juridique française pour mener à bien leur projet réformateur. J'insisterai davantage sur ce point que ne le fait Brown. Alors que ce dernier y voit avant tout un instrument efficace de résistance au colonialisme, j'y verrais personnellement d'abord le produit d'une relation asymétrique faisant apparaître le recours au transfert juridique comme une nécessité unilatérale. C'est bien parce qu'il y avait présence coloniale (ou menace dans d'autres contextes) qu'il y a eu recours au droit d'un colonisateur - fût-il autre que celui auquel on tentait de s'opposer. En d'autres termes, l'opération de transfert de technologie juridique n'a pas répondu à un processus de transformation endogène, mais à des questions posées par l'impérialisme de puissances étrangères. Ceci ne préjuge évidemment pas, dans un second temps, de l'efficacité du transfert dans la résistance à cet impérialisme et dans le projet de construction nationale. Simplement, je ne pense pas qu'on puisse affirmer que l'attractivité du modèle juridique d'importation n'a tenu qu'aux moyens accrus de contrôle, de centralisation et de pénétration qu'il offrait (Brown, 1995, p.116); son attractivité tenait également à sa nature occidentale, parce que l'Occident était tenu pour le modèle par excellence de pareille efficacité et que c'est l'expérience coloniale qui avait amené à s'en convaincre.

\section{Sharî'a, homologie structurelle et translation}

On ne peut donc limiter l'explication du succès de l'opération de transfert juridique au seul rapport asymétrique entre importateur et exportateur et il faut y voir également le résultat de son instrumentalisation par les élites réformatrices locales. J'ai déjà fait valoir l'homologie structurelle des systèmes juridiques importateur et exportateur. Il me semble à présent justifié de montrer à quel point l'existence d'une référence religieuse explicite dans le système importateur ne constitue pas un obstacle dirimant 
au processus de transfert. Cela tient sans doute à l'homologie structurelle des systèmes juridique et religieux. Une fois celle-ci expliquée, il me semble possible de ne plus voir le recours à la référence religieuse dans un cadre juridique positif sous l'angle de l'acrobatie incongrue, mais bien sous celui d'un simple mécanisme de translation.

Une première analogie peut être établie entre la relation au texte de droit et la relation au texte religieux. Droit et religion sont traversés par cette double tendance à monopoliser l'interprétation du texte et à le pétrifier. Il s'agit, d'une part, de postuler son adaptabilité parfaite, pour autant que soit respecté un appareil procédural d'interprétation, et, d'autre part, de postuler son immuabilité. L'un et l'autre vont d'ailleurs de pair, l'interprétation prétendant généralement garantir la littéralité. De plus, dans les deux cas, ce qui est visé, c'est l'assurance d'une situation monopolistique : monopole de l'interprétation et monopole du contrôle du respect de la lettre. Droit et religion semblent, à cet égard, traversés par les mêmes contraintes discursives. L'analogie des ordres théologique et juridique peut certainement être poussée plus loin, et ce à au moins trois niveaux.

On est d'abord redevable aux travaux de Pierre Legendre d'avoir identifié l'origine canonique et scolastique du droit dit moderne, dont on a déjà démontré l'exportation, «avec armes et bagages », en Égypte. L'analyse des langages du droit et de la religion permet enfin d'identifier leur commune performativité.

Il est inutile de revenir sur la démonstration faite par Legendre de la structure étroitement religieuse du droit occidental, que ce soit dans son histoire ou dans ses principes référenciels. On lui est ainsi redevable de nous avoir fait la démonstration de la constante référence à Dieu qui sous-tend le droit et son évolution, au moins dans l'espace occidental, parallèlement à l'affirmation de la forme étatique de gestion du politique. Le travail reste à faire pour la tradition islamique - et encore faudrait-il pouvoir définir ce que l'on entend par cela -, qui montrerait à quel point la constitution d'un corpus de droit, le figh, a procédé d'une manière relativement analogue à celle des droits romain et canon, instituant à la fois le Texte et ses glossateurs, prétendant à la fixité de la référence littérale tout en autorisant la quasi infinie variation de son interprétation et, surtout, assurant la reproduction d'une hiérarchie de pouvoir. On a tenté ailleurs d'esquisser la voie à suivre pour une telle investigation en s'attachant au fait politique coranique et islamique puis en faisant le détour par ses énonciateurs/ interprètes (Dupret, 1997 et à paraître).

L'analogie que l'on prétend pouvoir établir entre les discours juridique et religieux s'étend également à la nature même du langage que tous deux produisent.

On fait ici référence à la théorie de la structure performative de certaines formes linguistiques. En une formule serrée, l'on dira que le performatif est ce mode de discours qui fait du dire un acte efficace. S'agissant du droit, on remarquera sa capacité à influer sur le réel. Ainsi, à la suite de Clifford Geertz, il faut relever que «la pensée juridique est constructive de réalités sociales plutôt qu'elle n'est un simple reflet " (Geertz, 1986, p. 287). Le droit, en tant qu'il est une représentation du réel, exerce une influence sur la pratique de ce réel, lui-même à nouveau producteur de représentations, etc. Il en résulte un style performatif, prenant généralement la forme d'une prétention verbale ayant vocation à "réussir». Le langage de la foi peut également être considéré en tant que langage de la parole. Les énoncés religieux sont indissociables de leur énonciation et de l'effectivité de leur énonciation. Cela se manifeste, entre autres, dans la révélation en tant qu'énonciation divine. La logique 
performative du langage religieux est donc attestée, qui consiste à « faire ce que l'on dit dans l'acte même de le dire ».

Homologie structurelle et homologie linguistique, voilà certainement des arguments justifiant pleinement que le recours au répertoire du droit islamique par le juge égyptien ne puisse pas être compris en simples termes de contradiction. On remarquera, en toute hypothèse, que de nombreux exemples permettent d'illustrer l'étroite parenté des énoncés faisant appel aux deux répertoires juridiques positif et islamique.

La facilité de la conjonction des deux répertoires trouve à s'illustrer jusque dans leurs postulats interprétatifs. On ne prendra qu'un seul exemple, parmi de nombreux possibles. Il a été fait allusion plus haut à la doctrine du « sens clair » que fonde l'image du législateur rationnel. En vis-à-vis, l'extrait d'un arrêt récent de la Haute Cour constitutionnelle égyptienne développant la notion de «disposition de la sharî́a de signification et d'interprétation absolues » devrait se révéler significatif.

«Attendu que ce que la Constitution a stipulé en son article 2, après son amendement de 1980, quant au fait que les principes de la sharî́a islamique sont la source principale de la législation, porte entièrement sur une limitation à laquelle doit se conformer le pouvoir législatif pour les législations promulguées après l'entrée en vigueur de l'amendement constitutionnel précité, au titre desquelles les règles de la loi 100/1985 portant amendement de certaines dispositions des lois de statut personnel. Il n'est donc pas permis à un texte législatif de contredire les règles de la sharî'a (Légales) dont la filiation et l'interprétation sont concluantes, eu égard au fait que ces règles sont les seules pour lesquelles le raisonnement interprétatif (ijtihâd) est impossible, dans la mesure où elles représentent, dans la sharî'a islamique, les principes supérieurs et les fondements fixes qui ne supportent ni commentaire ( $\left.t a^{\prime} w \hat{l} l\right)$ ni permutation (tabdîl). On ne peut de plus s'attendre à ce que leur signification (mafhûm) se modifie en fonction du changement de temps et de lieu. Dès lors, elles sont réfractaires à tout amendement et il n'est pas permis d'en sortir ou d'en infléchir le sens. L'autorité de la Haute Cour constitutionnelle se limite en l'espèce à contrôler que l'on s'y tienne et qu'elles l'emportent sur toute règle légale qui les contredirait. Cela signifie que l'article 2 de la Constitution fait prévaloir sur ces règles les dispositions de la sharî'a dans leurs fondements et leur principes supérieurs. Elles constituent dès lors le cadre général et les structures authentiques dont les exigences s'imposent sans cesse, en ce qu'elles font obstacle à la fixation de n'importe quelle règle juridique qui leur serait contraire, sans quoi ceci serait considéré comme préjudiciable et attentatoire à ce que l'on sait nécessairement de la religion (ses postulats, mâ 'ulima bi-l-darûra). Il n'en va pas de même des règles autorisant le raisonnement individuel (ahkâm dhanniyya), qui sont relatives aussi bien dans leur filiation que dans leur signification, ou dans les deux. Cela signifie que le rôle de l'effort interprétatif se limite à elles et ne s'étend pas à d'autres. Il s'agit des règles qui sont, par nature, évolutives et qui se modifient par le changement de temps et de lieu, afin d'en garantir la souplesse et le dynamisme et pour faire face aux occurrences, dans leur disparité, en organisant les affaires des gens ('ibâd) d'une manière qui satisfasse leurs intérêts considérés comme légitimes, et pour de plus ne pas annuler leur mouvement dans la vie, dans la mesure où cet effort interprétatif est continuel et survient dans le cadre des fondements universels de la sharî'a sans les outrepasser, tout en se conformant à ses principes établis, en recherchant les méthodes de preuve en fonction des règles pratiques et des règles organisant ses différentes branches et en garantissant la préservation des buts généraux de la sharî'a, avec la préservation de la religion, de l'intégrité physique, de la raison, de la vertu et des biens que cela comporte. » (HCCE, 1994)

À la lecture de cet extrait, il apparaît que l'existence est très explicitement postulée. Je n'ai pas pu trouver une référence explicite à ces catégories des règles de signification et 
d'interprétation absolues et des règles découlant du raisonnement individuel. On peut probablement y voir l'indice que le juge égyptien fonctionne, quand il a recours au répertoire islamique, sur le mode $\mathrm{du}$ " comme si » le droit (islamique) était cohérent, transparent et non lacunaire (Lenoble et Ost, 1980). Ce qui est frappant par ailleurs, c'est l'importance du travail interprétatif, l'ijtihâd, que le juge constitutionnel égyptien s'arroge le droit de récupérer (même s'il l'attribue au législateur) et dont il fixe les modalités en interprétant la notion de travail interprétatif.

\section{Transfert et standards juridiques ; postulat d'identité de la sharîa}

Empruntée au langage ordinaire, la notion de standard, qui contient l'idée de «modèle conforme à la normale ", semble correspondre, dans le langage juridique, au «besoin d'isoler une certaine catégorie d'expressions normatives caractérisées par l'absence de toute prédétermination et l'impossibilité de les appliquer sans procéder au préalable à une appréciation ou une évaluation, c'est-à-dire en plaçant le fait auquel on les rapporte sur une échelle de valeurs " (Orianne, 1993, p. 581). Le modèle devient sans doute, en matière juridique, hypothétique à construire, quoiqu'en termes de représentations de ceux qui y ont recours, il paraisse davantage préconstitué. Toujours est-il que l'utilité du standard est d'ouvrir, dans le champ normatif, "un espace d'indétermination" autorisant celui qui doit appliquer la règle à davantage d'appréciation de l'état d'une chose, de ses qualités ou d'un comportement. Tout ceci pour dire qu'on peut se demander si la référence à la sharîa n'est pas devenue, dans le contexte juridique et judiciaire égyptien, un standard juridique parmi d'autres.

Nous affirmions plus haut que le recours au répertoire du droit islamique par le juge égyptien ne pouvait pas être compris en termes de contradiction. Il est certainement plus fécond de considérer, au vu de ce qui vient d'être dit, que le juge valorise un standard juridique face à un ou plusieurs autres standards que l'institution dominante tente d'accréditer comme étant seuls valides, alors même que son environnement le met en présence d'autres standards validés par d'autres réseaux de légitimation.

En Égypte, un certain type de juridicité transférée semble avoir bien pris racine. Il reste à rendre compte, dans ce cadre, de la place et de la fonction que la sharî'a a pu occuper. À cet égard, l'analyse de Massaji Chiba sur le postulat d'identité du droit indigène et sur sa fonction dans la transplantation juridique est éclairante (Chiba, 1986). Chiba fait d'abord le constat qu'un certain nombre de principes de droit positif ne peuvent être compris et ne trouvent pas à s'appliquer (du moins de manière autonome), dans les traditions juridiques non occidentales, sans qu'il ne soit fait recours à ce qu'il appelle des « compléments fonctionnels ». Pour pouvoir opérer, ces compléments fonctionnels doivent prendre la forme d'un cadre de référence permettant à certaines normes socioculturelles de fonctionner de pair avec le cadre légal établi (droit positif). Ceci implique, naturellement, d'avoir sur le droit un regard anthropologique reconnaissant la pertinence de la théorie du pluralisme juridique. À ce stade, Chiba construit son analyse sur trois dichotomies : droit officiel/droit non officiel; règle légale/postulat juridique (ou règle positive/valeur "postulative »); droit indigène/droit transplanté4. Dans un contexte de transplantation juridique, le droit non officiel (généralement le droit indigène) devient le véhicule par lequel, sous forme de postulat juridique (ou éventuellement de règle juridique), les compléments fonctionnels trouvent à remplir leur rôle. Les postulats juridiques proposent ainsi la traduction juridique des 
compléments fonctionnels dont la formulation est d'ordre social. La notion de " postulat d'identité du droit indigène " vise ce postulat juridique de base qui permet à un groupe de maintenir, dans le droit, ce qu'il se représente de son identité culturelle.

L'existence de postulats juridiques, en ce compris les postulats d'identité, est attestée dans l'ensemble des traditions juridiques. Il ne s'agit de rien d'autre que ce que précédemment on appelait standard juridique. Ce recours au standard juridique relève d'un passage de la norme entendue au sens statistique (ou ce que l'acteur considère relever de la norme statistique) à la norme entendue au sens juridique et contraignant. Si l'on reprend la distinction de Carol Greenhouse (1982), on sera enclin à dire que les postulats juridiques (dont celui d'identité) appartiennent à l'ordre de la justification davantage qu'à l'ordre de la prescription, c'est-à-dire de la règle de droit à proprement parler. Tel est probablement le cas de la sharî́a dans le contexte égyptien qui nous occupe. Cela apparaît en tout cas très explicitement dans l'échange de propos que nous avons eu avec A. W., avocat et ancien magistrat, en juin 1994.

«B.D.: Quand j'ai rencontré B.I. (magistrat égyptien), il m'a dit que ce qui manquait, ce n'était pas les textes, mais la référence, le texte de référence. Les textes appliquent la sharîa, mais on ne trouve pas cette référence globale.

«A.W.: Oui, c'est vrai. D'où vient ce cadre référentiel? Il faudrait que ce soit l'interprétation des textes et leur application en faisant référence aux fondements de l'islam. Si nous trouvions ce cadre référentiel présent, $90 \%$ de nos difficultés seraient résolues.»

Le postulat d'identité se caractérise par un contenu éminemment variable et son efficacité n'est garantie que par sa plasticité. Celle-ci procède de deux caractéristiques : d'une part, le postulat tend à circonscrire le ressort d'une collectivité et donc à déterminer les critères d'appartenance à celle-ci ; d'autre part, il tend à symboliser un système de valeurs particulier, donnant ainsi aux membres de la collectivité le référent de leur identité et de leur appartenance (Chiba, 1986, p. 41). On en vient ainsi à l'idée d'un droit participant à la définition de l'identité du groupe.

\section{BIBLIOGRAPHIE}

ABÛ TÂLIB S., 1994, «La sharî'a islamique et le droit positif dans les pays arabes : histoire et prospective ", dans B. BOTIVEAU et N. ABD AL-FATTAH, Politiques législatives:

Égypte, Tunisie, Algérie, Maroc, Le Caire, Cedej.

BOTIVEAU B. :

-1989, «Islamiser le droit? L'exemple égyptien », Maghreb Machrek, n 126, p. 5-24.

-1993, Loi islamique et droit dans les sociétés arabes. Mutations des systèmes juridiques du Moyen-Orient, Paris, Karthala.

BROWN N. J., 1995, « Law and Imperialism: Egypt in Comparative Perspective », Law\& Society Review, $\mathrm{n}^{\circ}$ 29/1, p. 103-125. 
CHIBA M., 1986, « The Identity Postulate of Indigenous Law and its Function in Legal Transplantation », in SACK P. and MINCHIN E. (eds.), Legal Pluralism, Proceedings of the Camberra Law Workshop VII, Camberra, Australian National University.

CROMER (Earl of), 1911, Modem Egypt, (2nd ed.) London, Macmillan.

DUPRET B. :

- 1995, « Entre le droit et la Loi. Le juge et le jeu de la normalisation islamique du droit positif », Droit et Cultures, $n^{\circ} 30$, p. 47-64.

- 1997, « Interpreting Political Islam. A Diachronical Approach to the Koran », in M. SCHMIEGELOW (ed.), Democracy in Asia, Frankfurt, Campus Verlag et New York, St Martin's Press.

- à paraître dans les annales HSS, « L'historicité de la norme », Du positivisme de l'islamologie juridique à l'anthropologie de la norme islamique.

GEERTZ C., 1986, Savoir local, savoir global. Les lieux du savoir, Paris, PUF.

GHURÂB M. 'A. H., 1986, Jugements islamiques contredisant les lois positives (en arabe), Le Caire, Dâr al-i'tisâm.

GOLDBERG J., 1997, « L'Europe au-delà de l'Europe : réflexions sur l'entrée de l'Egypte dans la famille française du droit », dans G. BOËTSCH, B. DUPRET et J.-N. FERRÉ, Droits et sociétés dans le monde arabe, Aix-en-Provence, Presses de l'Université d'Aix-Marseille.

GREENHOUSE C. J., 1982, « Looking at culture, looking for rules », Man, n 17, p. 58-73.

GRIFFITHS J.:

- 1978, « Is Law Important? », New York University Law Review, n 54 p. 339-374.

-1986, « What is Legal Pluralism? », Journal of Legal Pluralism, n²4, p. 1-55.

-1990, « Legal Pluralism and the Social Working of Law » (non publié).

HILL E., 1987, « Al-Sanhuri and Islamic Law », Cairo Papers in Social Science, n 10/1.

JACQUEMOND R., 1988, «Égypte : la Haute Cour constitutionnelle et le contrôle de constitutionnalité des lois (1979-1987) », Annuaire international de justice constitutionnelle, $\mathrm{n}^{\circ} 4$, p. 271-295.

LENOBLE J. et OST F., 1980, Droit, mythe et raison. Essai sur la dérive mythologique de la rationalité juridique, Bruxelles, Publications des Facultés universitaires Saint-Louis.

ORIANNE P., 1993, « Standard juridique », dans ARNAUD A.-J., Dictionnaire encyclopédique de théorie et de sociologie du droit, Paris, LGDJ.

REID D., 1981, Lawyers and Politics in the Arab World, Minneapolis-Chicago, Bibliotheca Islamica.

ROULAND N., 1988, Anthropologie juridique, Paris, PUF.

ZIADEH F, 1968, Lawyers, theRuleofLawand Liberalism in Modem Egypt, Stanford, Hoover Institution. 


\section{NOTES}

1. La description qui suit s'inspire très largement du chapitre consacré par Enid Hill, dans son travail sur le célèbre juriste égyptien 'Abd al-Razzâq al-Sanhûrî (1895-1971), à l'émergence d'un système juridique moderne en Égypte.

2. Entendons par là le droit produit par les institutions autorisées de l'État. Il n'est en toute hypothèse pas question d'ignorer tout l'acquis des théories du pluralisme juridique (lire à cet égard Rouland, 1988, p. 74-98 et 102-111), en tête desquelles il faut citer les travaux autorisés de J. Griffiths (1978,1986 et 1990), mais simplement de relever que, si l'objet de la sociologie du droit n'est effectivement pas tant la loi, en tant que catégorie distincte, que la notion de contrôle social et si, dans cette perspective, ce que les juristes et souvent lessociologues du droit appellent « loi » correspond en fait à une forme fortement (mais pas exclusivement) spécialisée de contrôle social (Griffiths, 1990, repris dans Dupret, 1995), il n'en demeure pas moins que cette «forme spécialisée de contrôle social » qu'est le droit de l'État continue de vivre et de se développer, de même que l'institution judiciaire qui l'accompagne. Il est dès lors justifié de traiter, dans une anthropologie juridique faisant $d u$ pluralisme un principe fondamental, des normes et des comportements juridiques externes et internes à l'État. Cette distinction est, de plus, largement artificielle, dès lors que ces différents champs juridiques sont à la fois imbriqués et exercent l'un sur l'autre une action majeure.

3. «S'il y a bien eu «transfert de technologie juridique », cette familiarité avec le droit français concerne surtout la minorité des professeurs de droit. Leur influence dans la production de la doctrine juridique est certes prépondérante, mais la création jurisprudentielle est, quant à elle, le produit de conditions nationales. Formellement, l'enseignement du droit s'est doté d'une pédagogie comparable à celle des facultés françaises, mais il en diffère beaucoup quant à ses contenus. L'enseignement en arabe se réfère à des concepts qui sont parfois d'origine étrangère, mais qui, souvent, ont été forgés par la culture juridique des pays concernés: droit écrit, interprétations doctrinales, jurisprudences nationales, fiqh islamique. » (Botiveau, 1993, p. 168)

4. «Le «droit officiel » est le système légal et ses composantes sanctionnés par l'autorité législative d'un pays, qui est typiquement représentée par le «droit étatique » dans le monde moderne. Le « droit non officiel » est le système légal (ou certaines de ses composantes) qui n'est pas sanctionné officiellement par une autorité légitime quelconque, mais qui est appliqué en pratique par le consensus général d'un cercle déterminé de gens, que ce soit à l'intérieur ou audelà des limites d'un pays, qui exerce une influence distincte sur l'effectivité du droit officiel en y suppléant, s'y opposant, le modifiant ou l'invalidant, en tout ou en partie.» (Chiba, 1986, p. 39-40). «La «règle juridique» est l'expression verbale formalisée d'une disposition légale particulière désignant certains modèles spécifiques de comportement. Les "postulats légaux » sont des valeurs particulières ou des idées spécifiquement connectées à un système juridique particulier qu'elles justifient et qui peuvent donc être utilisées pour critiquer et revoir ses règles juridiques. » (Chiba, 1986, p. 40). « Le « droit indigène » est le droit qui trouve son origine dans la culture originelle d'un peuple. Le "droit transplanté » est le droit transplanté d'une culture à d'autres, que ce soit par réception volontaire ou par imposition.» (Chiba, 1986, p. 40).

\section{INDEX}

Mots-clés : droit, histoire du droit, sharî’a 
AUTEUR

BAUDOUIN DUPRET

Cedej 